\title{
Prophylactic intracameral levofloxacin in cataract surgery - an evaluation of safety
}

This article was published in the following Dove Press journal:

Clinical Ophthalmology

\author{
Cesar Ramon G Espiritu',2,* \\ Joanne G Bolinao $1, *$
}

'American Eye Center, Mandaluyong, ${ }^{2}$ Department of Ophthalmology, Manila Doctors Hospital, Manila, Philippines

*The authors contributed equally to this work
Correspondence: Cesar Ramon G

Espiritu

Department of Ophthalmology, Manila

Doctors Hospital, 667 UN Avenue,

Ermita, Manila 1000, Philippines

Tel +6325272260

Email espiritueyemd@mac.com
Purpose: To evaluate posterior and anterior segment safety of an intracameral injection of levofloxacin $0.5 \%$ ophthalmic solution as prophylaxis for patients undergoing cataract extraction and intraocular lens implantation.

Setting: This study was conducted at Manila Doctors Hospital, Ermita, Manila, Philippines. Design: This was a prospective interventional study.

Methods: Eyes undergoing standard phacoemulsification cataract surgery with intraocular lens implantation were treated with intracameral levofloxacin $0.5 \%$ at the conclusion of surgery. Safety parameters, including best-corrected visual acuity (BCVA), endothelial cell counts, anterior chamber cells and flare, and central foveal thickness, were evaluated preoperatively and at 1 day and 1 week postoperatively.

Results: A total of 50 eyes of 50 patients were included in the analysis. At 1 week postoperatively, all eyes demonstrated BCVA of 20/30 or better and 19 eyes (38\%) achieved BCVA of $20 / 20$ or better. On the first postoperative day, no corneal edema was observed, and trace to +2 cells and flare in the anterior chamber were noted in all eyes. After 1 week, all eyes had a quiet anterior chamber and endothelial cell counts decreased by an average of $225 \mathrm{cells} / \mathrm{mm}^{2}$, which was marginally significant $(p=0.0525)$ when compared to other time points. Optical coherence tomography results showed no statistically significant differences between central foveal thickness measurements before and after surgery. There were also no statistically significant differences in preoperative and postoperative pachymetry. No study-related adverse events occurred.

Conclusion: There were no safety concerns associated with intracameral injection of levofloxacin $0.5 \%$, prophylactically, following cataract surgery. Further study is required to demonstrate effectiveness in endophthalmitis prevention.

Keywords: intracameral levofloxacin, endophthalmitis prevention, phacoemulsification, cataract surgery, intraocular surgery, prophylaxis

\section{Introduction}

Cataract surgery remains the most commonly performed ocular procedure worldwide. The advances in technology and improvement in surgical techniques have resulted in better visual outcomes, thereby increasing patients' expectation of optimal results. Although the incidence of endophthalmitis remains low following routine cataract extraction, the risk still exists as a potentially blinding, albeit preventable, complication. Surgeons are mindful of the importance of careful standardized antiseptic techniques, including the use of topical povidone-iodine using the $5 \%$ solution and povidone-iodine scrub of the ophthalmic area with $10 \%$ solution. Common standard practices also include administration of preoperative and postoperative topical antibiotics. 
Antibiotic prophylaxis for the prevention of postoperative ocular infections has been found to be beneficial. A survey conducted in 2007 by the members of the American Society of Cataract and Refractive Surgery (ASCRS) suggested a strong preference for perioperative topical antibiotic prophylaxis. ${ }^{1}$ Most respondents favored the newer generation fluoroquinolones; however, results also demonstrated a tangible concern with the use of reconstituted antibiotic preparations. ${ }^{1}$ Although some orally administered antibiotics can achieve intraocular concentrations via systemic delivery, others do not effectively penetrate the eye. Intracameral antibiotic administration is the most direct route of delivery to the site of potential infection.

In the multicenter European Society of Cataract \& Refractive Surgeons (ESCRS) study, intracameral cefuroxime was injected at the end of surgery and topical levofloxacin was given immediately preoperatively (within 1 hour of surgery) and up to 15 minutes following surgery in 16,603 participants. $^{2}$ The results showed that postoperative endophthalmitis at 6 weeks was significantly reduced for eyes that received intracameral cefuroxime injections, with or without topical levofloxacin, compared with eyes that received no prophylaxis. ${ }^{2}$ The study design and large sample size were able to isolate and confirm that intracameral injection was the key factor in the demonstrated reduction of endophthalmitis from what was typically expected. Additionally, a recent report from India has demonstrated a reduced risk of endophthalmitis from $0.08 \%$ among patients who had not received intracameral antibiotics down to $0.02 \%$ among those who had received intracameral antibiotics. ${ }^{3}$

The value of perioperative intracameral injection of antibiotic at the end of cataract surgery is becoming increasingly recognized as a method to provide immediate, sustained, high concentration of antibiotic over a period of time. Although perioperative intracameral antibiotic administration was controversial in the past, a growing body of evidence has demonstrated a significant reduction in the incidence of endophthalmitis when this technique is used..$^{2-4}$ In addition, the safety of intracameral injection of antibiotics following cataract surgery has been demonstrated by evaluating parameters, including anterior chamber reaction, corneal thickness, and corneal endothelial density. ${ }^{5}$

As an update to the 2007 survey, the results of the 2014 survey of ASCRS members showed that perioperative use of intracameral antibiotics as prophylaxis had increased to $50 \%$, an increase from $30 \%$ in $2007 .{ }^{6}$ In all, $84 \%$ of respondents also reported a preference for direct injection over irrigation of fluid. ${ }^{6}$ Additionally, postoperative intracameral injection, as a method of antibiotic administration at the conclusion of the surgery, increased to $36 \%$ from only $14 \%$ in $2007 . .^{1,6}$

As intracameral antibiotic injection is quickly being adopted as standard of care in many centers, the debate is now focused on which antibiotic is safest and most effective to choose for these patients. Cefuroxime, vancomycin, and moxifloxacin are the most widely used antibiotics associated with cataract surgery. However, cefuroxime and vancomycin are currently available in systemic preparations, which require reconstitution. Reports of ocular toxicity, following dilution errors, have raised concerns among surgeons. ${ }^{7,8}$ Additionally, some surgical centers, such as the one where this study was conducted, lack direct pharmacy access, which would make an antibiotic preparation that required no dilution or reconstitution ideal. The authors have reported their previous experience with intracameral moxifloxacin since 2006 and have noted no toxicities or safety concerns related to its use. ${ }^{5}$ The authors did, however, want to investigate whether other topical antibiotics were also safe for intracameral administration.

Levofloxacin, a broad-spectrum third-generation fluoroquinolone, was selected for this study for several important reasons. Levofloxacin was the topical agent used in the ESCRS study, it is commercially available in a selfpreserved $0.5 \%$ formula that requires no dilution and it has demonstrated susceptibility to both Gram-positive and Gram-negative organisms. Preclinical studies compared the toxicity of intracameral injection of cefazolin, levofloxacin, and moxifloxacin, and did not find any significant toxicity on the endothelial cells compared with the control group, concluding that intracameral injection of any of the studied antibiotics appeared to be safe for surgical prophylaxis. ${ }^{9}$

The purpose of this study was to evaluate posterior and anterior segment safety of an intracameral injection of levofloxacin $0.5 \%$ ophthalmic solution as prophylaxis for patients undergoing cataract extraction and intraocular lens implantation.

\section{Patients and methods}

This was a prospective, single-center, single-surgeon (CRGE) study of consecutive patients scheduled to have standard phacoemulsification cataract surgery. The inclusion criteria comprised patients between the ages of 55 and 85 years, inclusive, who were diagnosed with cataract opacities between NO2NC2 and NO4NC4, based on the Lens Opacities Classification System III (LOCS III). Patients were excluded from the study if they had been diagnosed with other ocular pathologies, other than cataract, such as glaucoma, 
retinopathy-maculopathy, and other media opacities (cornea or vitreous). Those patients with visual pathway problems that would prevent achievement of best-corrected visual acuity (BCVA) of 20/30 or better or those with uveitis, diabetes, corneal endothelial disease, pseudoexfoliation, systemic immunosuppressants, or anticoagulants were also excluded. Those eyes that experienced any intraoperative complications or a particularly difficult surgical procedure were excluded from the study as the outcomes of the safety parameters of levofloxacin may be erroneously influenced by these factors. This study was approved by the institutional review board of Manila Doctors Hospital. Patients provided signed informed consent to be included in this study.

Preoperative pupil dilation was achieved with a solution of tropicamide $0.5 \%$ and phenylephrine $\mathrm{HCl} 0.5 \%$ (Sanmyd-P; Santen Pharmaceutical Co., Ltd., Osaka, Japan). Preoperatively, patients also received one drop of topical $0.5 \%$ levofloxacin ophthalmic solution (Oftaquix; Santen Pharmaceutical) every 15 minutes for at least four doses. A drop of povidone iodine $0.5 \%$ was instilled in the cul-de-sac of the eye prior to surgery, and povidone iodine $10 \%$ paint was applied on the ophthalmic area before commencement of the procedure.

Standard phacoemulsification was performed by a single surgeon (CRGE) under topical anesthesia using proparacaine $0.5 \%$ (Alcaine; Alcon Laboratories, Inc., Fort Worth, TX, USA) with an additional intracameral injection of $1 \%$ preservative-free xylocaine. Surgery was performed through a 2.2-2.4 mm clear corneal incision on the temporal side and a side port $70^{\circ}$ apart. Sodium chondroitin sulfate-sodium hyaluronate gel (Viscoat; Alcon Laboratories, Inc.) was used during nuclear removal and sodium hyaluronate $1.0 \%$ gel (ProVisc; Alcon Laboratories, Inc.) for intraocular lens implantation. A single-piece hydrophobic acrylic monofocal intraocular lens (IOL) from a single manufacturer, AcrySof SN60AT, SN60WF or SN6AD3 (Alcon Laboratories, Inc.), was used for all subjects.

Using a tuberculin syringe, $0.3-0.5 \mathrm{~mL}$ of $0.5 \%$ levofloxacin ophthalmic solution was aspirated from a newly opened bottle. At the conclusion of cataract extraction, intraocular implantation, and viscoaspiration, the excess over $0.1 \mathrm{~mL}$ of the solution was discarded onto the ocular surface, and $0.1 \mathrm{~mL}$ solution containing $0.5 \mathrm{mg}$ levofloxacin was injected via a 27-gauge cannula through the side port into the capsular bag.

Postoperative medications included topical $0.5 \%$ levofloxacin and topical $1 \%$ prednisone acetate, both administered every 2 hours during the first 24 hours, followed by four times daily until the bottles were consumed, $\sim 3$ weeks after surgery.

The patients were examined before surgery and then at 1 day and 1 week after surgery. Evaluation of safety included anterior chamber reaction expressed in cells and flare as observed on biomicroscopy; BCVA using a Snellen chart; endothelial cell counts, expressed in cells $/ \mathrm{mm}^{2}$ of the central cornea measured using a noncontact specular microscope; and central foveal thickness in micrometers measured using the Zeiss Stratus OCT (Carl Zeiss Meditec AG, Jena, Germany). Central foveal thickness assessment was included to demonstrate any changes that may represent findings of cystoid macular edema.

Data were recorded and analyzed using analysis of variance (ANOVA). Null hypothesis was preoperative, and postoperative endothelial cell count and foveal thickness are equal. A $p$-value of $<0.05$ was considered significant.

\section{Results}

A total of 50 eyes of 50 patients, all of Filipino descent, were included in the analysis. There were 35 female patients and 15 males. The mean age at the time of surgery was 71 years (range 55-86 years). At 1 day postoperatively, all eyes demonstrated BCVA of 20/30 or better and 19 eyes (38\%) achieved BCVA of 20/20 or better. At 1 week postoperatively, all eyes demonstrated BCVA of 20/30 or better, and 32 eyes (64\%) achieved BCVA of 20/20 or better (Figure 1).

On the first day following surgery, trace to +2 cells and flare in the anterior chamber were noted in all eyes (Figure 2). No central corneal edema was observed in any eyes at day 1 . After 1 week, all eyes had a quiet anterior chamber, and endothelial cell counts decreased in 37 eyes

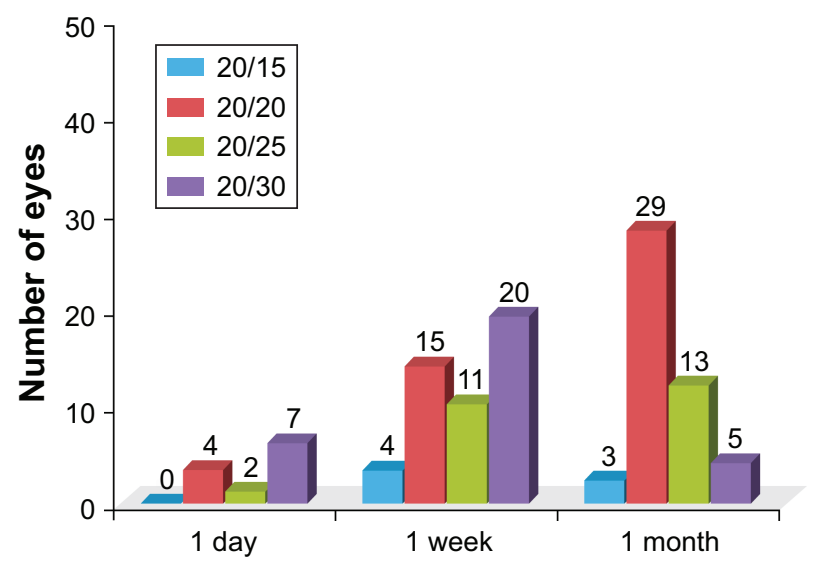

Figure I Postoperative visual acuity of patient population (BCVA). Abbreviation: BCVA, best-corrected visual acuity. 


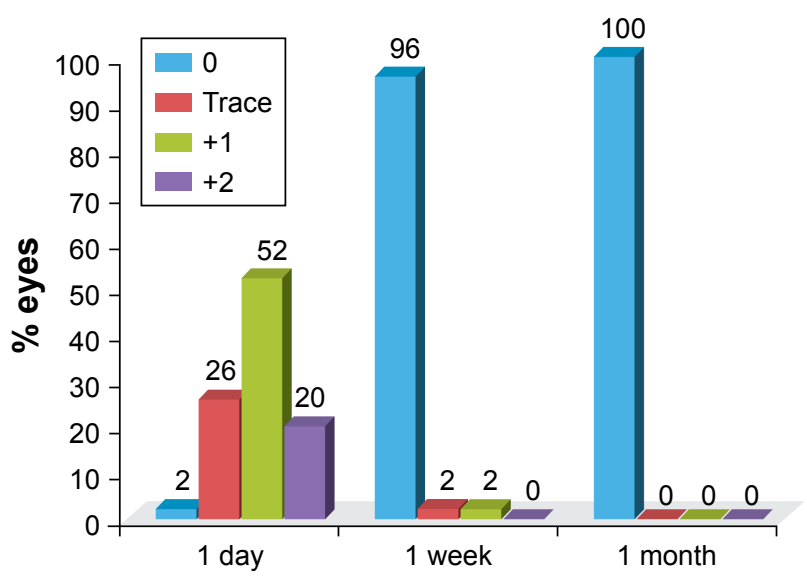

Figure 2 Anterior chamber reaction: postoperative endothelial cell count $\left(\right.$ cells $/ \mathrm{mm}^{2}$ ), illustrating the presence of corneal edema.

by a mean of 225 cells $/ \mathrm{mm}^{2}$, which when compared to other time points was marginally significant $(p=0.0525)$ using ANOVA. There is no evidence to demonstrate significant differences in preoperative and postoperative endothelial cell counts (Figure 3 ).

The mean preoperative pachymetry was $541 \mu \mathrm{m}$, while the mean postoperative pachymetry after 1 week was $563 \mu \mathrm{m}$, which was not a statistically significant finding $(p=0.0953$; Figure 4). OCT demonstrated no statistically significant differences $(p=0.3466)$ between central foveal thickness measurements before or after surgery (Figure 5). No studyrelated adverse events occurred.

\section{Discussion}

In the UK, a 2009 survey reported that $\sim 50 \%$ of providers use intracameral antibiotics following cataract surgery. ${ }^{10}$ Likely due to the ESCRS recommendations, an analysis of billing codes in France suggested that up to $80 \%$ of providers use intracameral antibiotics between 2005 and 2014. ${ }^{11}$ As can be inferred from the lower provider use but steady

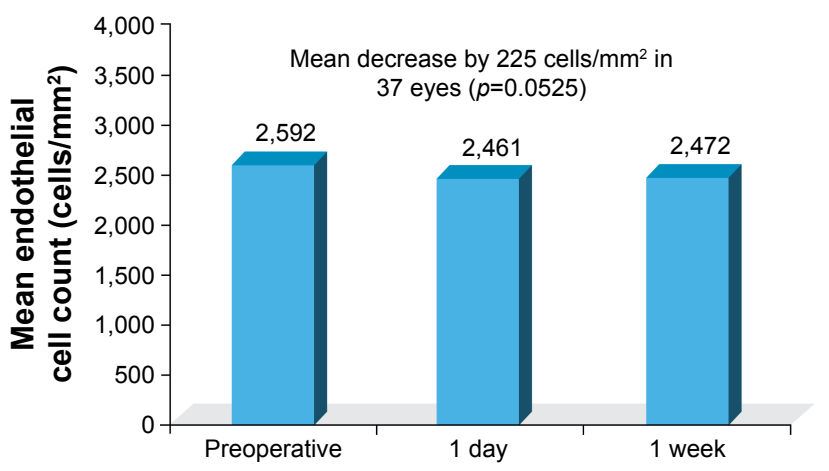

Figure 3 Comparison of mean endothelial cell count (cells $/ \mathrm{mm}^{2}$; preoperative vs postoperative).

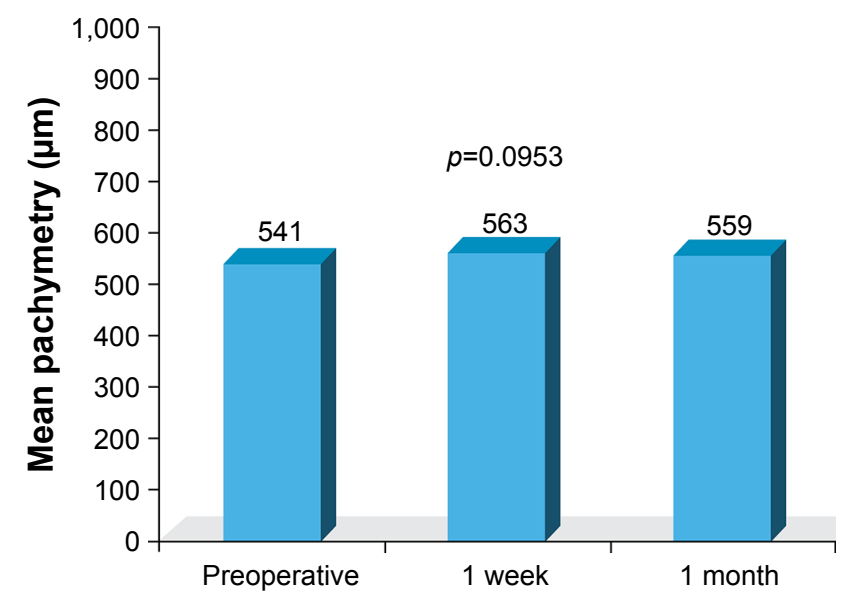

Figure 4 Comparison of mean pachymetry ( $\mu \mathrm{m}$; preoperative vs postoperative).

rise in uptake in the US - 30\% in 2007 and 50\% in $2014-$ skepticism of the safety and effectiveness of intracameral antibiotics is slowly being addressed. ${ }^{1,5}$ Although several studies of intracameral injection have reported reduced rates of endophthalmitis, compared with no antibiotic injection, the choice of antibiotic has varied, including moxifloxacin, vancomycin, and cefuroxime, and the ideal choice of antibiotic agent remains unclear., ${ }^{42}$ Additionally, identified barriers to provider uptake have included a lack of commercially available preparations and concerns regarding dilution errors and risk of contamination when compounding the drugs for required doses. Ophthalmologists in the US have indicated that intracameral antibiotic use would likely increase if a single-dose vial was commercially available. ${ }^{6}$

Intracameral cefuroxime has been the most extensively studied in terms of safety and efficacy, likely due to the results of the ESCRS study and the products' increased availability in Europe. A single-use vial of cefuroxime, which requires only reconstitution and not dilution, was approved in Europe in 2012. The ESCRS study observed

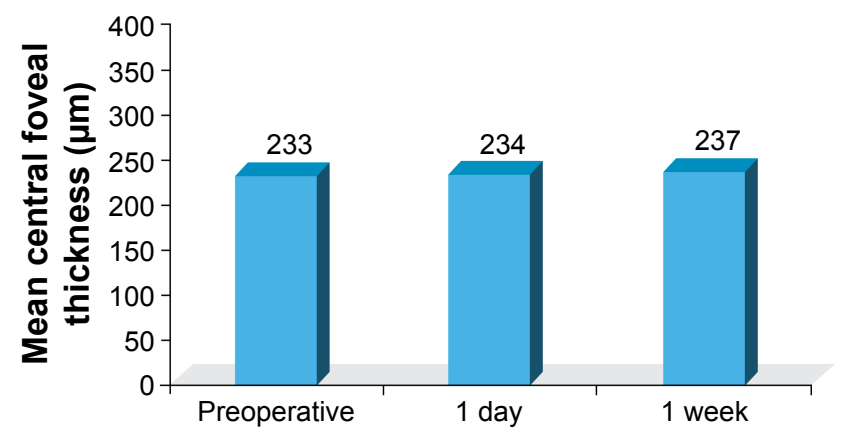

Figure 5 Comparison of mean central foveal thickness measurements $(\mu \mathrm{m}$; preoperative vs postoperative). 
a significant risk reduction of infection in eyes treated with intracameral cefuroxime. ${ }^{2}$ In their multicenter study, endophthalmitis occurred in $23(0.335 \%)$ of 6,862 patients who did not receive cefuroxime compared with $5(0.073 \%)$ of 6,836 patients who received intracameral cefuroxime. Investigators in Sweden reported extremely low rates of postoperative endophthalmitis $(0.029 \%)$ in the presence of intracameral cefuroxime, and in Portugal, a center reduced endophthalmitis from $0.26 \%$ to $0.0 \%$ after introduction of the ESCRS intracameral protocol. ${ }^{13,14}$ In the US, Shorstein et $\mathrm{a}^{15}$ reinforced these observations when their study of 16,264 eyes from a center in Northern California concluded a decline in the incidence of endophthalmitis after adopting intracameral antibiotic injection of cefuroxime, moxifloxacin, or vancomycin.

Moxifloxacin (Vigamox; Novartis International AG, Basel, Switzerland) is available as a self-preserved commercial ophthalmic formulation that requires no special preparation for intracameral delivery, thus reducing the risk of dilution errors. It is less extensively studied, but it is a fourth-generation fluoroquinolone that covers a broad spectrum of organisms and hence is effective in preventing postsurgical infection. The safety of intracameral moxifloxacin was confirmed in 2007 by the authors of the current article. ${ }^{5}$ We injected $0.1 \mathrm{~mL}$ of moxifloxacin $0.5 \%$ solution or an equivalent of $0.5 \mathrm{mg}$ moxifloxacin into the capsular bag at the conclusion of surgery. Intracameral moxifloxacin was nontoxic by parameters of visual rehabilitation, anterior chamber reaction, pachymetry, and corneal endothelial cell density. These results align with those from the current study, as no toxicities were observed with the use of intracameral levofloxacin.

Reservations exist toward the routine prophylactic use of fourth-generation fluoroquinolones, such as moxifloxacin. Some health care providers prefer to withhold moxifloxacin unless required for specific treatment, and infectious disease authorities have expressed concern related to the growing resistance to antibiotics and discourage its use for prophylaxis.

Schimel et al ${ }^{16}$ reported that for Gram-positive organisms, there was only $47 \%$ susceptibility to moxifloxacin, and they did not note the susceptibility of Gram-negative organisms. They did, however, find that Gram-positive organisms had a $58.5 \%$ susceptibility rate to levofloxacin, while Gramnegative organisms demonstrated $100 \%$ susceptibility. It is important to recognize that although Gram-positive organisms are still the most common, series have been reported, including a Singapore study that evaluated Asian eyes, that
Gram-negative organisms comprise a bigger proportion of isolates $(25 \%)$ compared to Western studies. ${ }^{17}$

Levofloxacin, a broad-spectrum third-generation fluoroquinolone, was selected for this study for several important reasons. Levofloxacin was the topical agent used in the ESCRS study; it is commercially available in a self-preserved $0.5 \%$ formula, which requires no dilution, and as noted earlier, it demonstrated susceptibility to both Gram-positive and Gram-negative organisms. Preclinical studies by Kim et $\mathrm{al}^{9}$ compared the toxicity of intracameral injection of cefazolin, levofloxacin, and moxifloxacin, and did not find any significant toxicity on the endothelial cells compared with the control group. They concluded that intracameral injection of any of the studied antibiotics appears to be safe for surgical prophylaxis. Similarly, our study also did not observe any toxicities of intracameral levofloxacin.

We found there to be a paucity in the published literature of studies evaluating intracameral injection of levofloxacin and feel that it should be a topic of larger comparative studies.

\section{Conclusion}

The study demonstrated that there were no safety concerns associated with intracameral injection of levofloxacin $0.5 \%$, prophylactically, following cataract surgery. In our study of 50 eyes, there were no concerns related to corneal edema, endothelial cell loss, anterior chamber reaction, and central foveal thickness. Further study is required to demonstrate the effectiveness in endophthalmitis prevention.

\section{Acknowledgment}

We are thankful for the financial support provided by Santen Pharmaceutical Co., Ltd., for $0.5 \%$ levofloxacin commercial preparation and fees for diagnostic examinations, including biometry, pachymetry, specular microscopy, and OCT.

\section{Disclosure}

The authors have no proprietary interest in the materials used in this study and report no conflicts of interest in this work.

\section{References}

1. Chang DF, Braga-Mele R, Mamalis N, et al. ASCRS cataract clinical committee. J Cataract Refract Surg. 2007;33(10):1801-1805.

2. Barry P, Seal DV, Gettinby G, Lees F, Peterson M, Revie CW; ESCRS Endophthalmitis Study Group. ESCRS study of prophylaxis of postoperative endophthalmitis after cataract surgery: preliminary report of principal results from a European multicenter study. J Cataract Refract Surg. 2006;32(3):407-410.

3. Haripriya A, Chang DF, Namburar S, Smita A, Ravindran RD. Efficacy of intracameral moxifloxacin endophthalmitis prophylaxis at Aravind Eye Hospital. Ophthalmology. 2016;123(2):302-308. 
4. Gower EW, Lindsley K, Tulenko SE, Nanji AA, Leyngold I, McDonnell PJ. Perioperative antibiotics for prevention of acute endophthalmitis after cataract surgery. Cochrane Database Syst Rev. 2017;2:CD006364.

5. Espiritu CR, Caparas VL, Bolinao JG. Safety of prophylactic intracameral moxifloxacin $0.5 \%$ ophthalmic solution in cataract surgery patients. J Cataract Refract Surg. 2007;33(1):63-68.

6. Chang DF, Braga-Mele R, Henderson BA, et al; ASCRS Cataract Clinical Committee. Antibiotic prophylaxis of postoperative endophthalmitis after cataract surgery: results of the 2014 ASCRS member survey. $J$ Cataract Refract Surg. 2015;41(6):1300-1305.

7. Delyfer MN, Rougier MB, Leoni S, et al. Ocular toxicity after intracameral injection of very high doses of cefuroxime during cataract surgery. J Cataract Refract Surg. 2011;37(2):271-278.

8. Olavi P. Ocular toxicity in cataract surgery because of inaccurate preparation and erroneous use of $50 \mathrm{mg} / \mathrm{ml}$ intracameral cefuroxime. Acta Ophthalmol. 2012;90(2):e153-e154.

9. Kim SY, Park YH, Lee YC. Comparison of the effect of intracameral moxifloxacin, levofloxacin and cefazolin on rabbit corneal endothelial cells. Clin Exp Ophthalmol. 2008;36(4):367-370.

10. Gore DM, Angunawela RI, Little BC. United Kingdom survey of antibiotic prophylaxis practice after publication of the ESCRS Endophthalmitis Study. J Cataract Refract Surg. 2009;35(4):770-773.

11. Creuzot-Garcher C, Benzenine E, Mariet AS, et al. Incidence of acute postoperative endophthalmitis after cataract surgery: a nationwide study in France from 2005 to 2014. Ophthalmology. 2016;123(7):1414-1420.
12. Packer M, Chang DF, Dewey SH, et al; ASCRS Cataract Clinical Committee. Prevention, diagnosis, and management of acute postoperative bacterial endophthalmitis. J Cataract Refract Surg. 2011;37(9): 1699-1714.

13. Friling E, Lundstrom M, Stenevi U, Montan P. Six-year incidence of endophthalmitis after cataract surgery: Swedish national study. J Cataract Refract Surg. 2013;39(1):15-21.

14. Beselga D, Campos A, Castro M, et al. Postcataract surgery endophthalmitis after introduction of the ESCRS protocol: a 5-year study. Eur $J$ Ophthalmol. 2014;24(4):516-519.

15. Shorstein NH, Winthrop KL, Herrinton LJ. Decreased postoperative endophthalmitis rate after institution of intracameral antibiotics in a Northern California eye department. J Cataract Refract Surg. 2013; 39(1):8-14.

16. Schimel AM, Miller D, Flynn HW Jr. Endophthalmitis isolates and antibiotic susceptibilities: a 10-year review of culture-proven cases. Am J Ophthalmol. 2013;156(1):50-52.

17. Tan CS, Wong HK, Yang FP. Epidemiology of postoperative endophthalmitis in an Asian population: 11-year incidence and effect of intracameral antibiotic agents. J Cataract Refract Surg. 2012;38(3): 425-430.
Clinical Ophthalmology

\section{Publish your work in this journal}

Clinical Ophthalmology is an international, peer-reviewed journal covering all subspecialties within ophthalmology. Key topics include: Optometry; Visual science; Pharmacology and drug therapy in eye diseases; Basic Sciences; Primary and Secondary eye care; Patient Safety and Quality of Care Improvements. This journal is indexed on Submit your manuscript here: http://www.dovepress.com/clinical-ophthalmology-journal

\section{Dovepress}

PubMed Central and CAS, and is the official journal of The Society of Clinical Ophthalmology (SCO). The manuscript management system is completely online and includes a very quick and fair peer-review system, which is all easy to use. Visit http://www.dovepress.com/ testimonials.php to read real quotes from published authors. 